\title{
ЭФФЕКТЫ РЕГУЛЯЦИИ И СТИМУЛЯЦИИ: ИНСТИТУЦИОНАЛЬНЫЕ МЕХАНИЗМЫ ВОЗНИКНОВЕНИЯ И РОЛЬ В ИННОВАЦИОННОМ РАЗВИТИИ ХОЗЯЙСТВЕННЫХ СИСТЕМ
}

\author{
Д.Ю. Миропольский, И.Б. Ломакина*
}

Исследовано влияние институтов плана и рынка на формирование эффектов регуляции и стимуляции на основе использования двухсекторной модели хозяйственной системы. Обоснована важность для инновационного развития сочетания положительных и отрицательных значений данных эффектов в конкретных условиях определенной национальной экономики. Определена и графически проиллюстрирована динамика эффектов при движении от плана к рынку и от рынка к плану.

Ключевые слова: институты плана, институты рынка, эффект регуляции, эффект стимуляции, инновационное развитие.

JEL-классификация: A10, E02, E11.

DOI: $10.46782 / 1818-4510-2020-2-132-142$

Материал поступил 24.04.2020 2.

\section{Инновационное развитие в хозяйственных системах рыночного и планового типов}

Индустриальная стадия характеризовалась, по меньшей мере, тремя особенностями. Во-первых, процесс воспроизводства осуществлялся, в основном, в рамках национальной экономики; во-вторых, научно-техническое развитие шло сравнительно медленными темпами; в-третьих, в большинстве отраслей существовала лишь одна ведущая технология. В этих условиях, основным средством поддержания национальной конкурентоспособности была способность концентрировать ресурсы на известных, заданных направлениях инновационного развития.

На информационной (постиндустриальной) ситуация кардинально меняется. Глобализация не позволяет замкнуть воспроизводство в масштабах национального хозяйства; научно-технический прогресс резко ускорился; вместо одного технологического решения существует от пяти до семи. Эта новая реальность сместила акцент в конкурентной борьбе государств с кон- центрации ресурсов на инновации на сами инновации. Ты не можешь быть лидером, заимствуя инновации, ты сам должен быть источником инноваций.

То, что в современных условиях статус и конкурентоспособность национальной экономики определяется степенью ее инновационности, стало общим местом в экономической науке (Беломестнов, 2019. С. 30; Бодрунов, 2018. С. 74; Глазьев, 2016. С. 233239; Шульце, 2017. С. 40-41). Посмотрим, как обстоят дела в данной области в России (табл. 1).

Таблица 1

Затраты на НИОКР в России, Китае и Германии, \% к ВВП

\begin{tabular}{|c|c|c|c|}
\hline \multirow{2}{*}{ Год } & \multicolumn{3}{|c|}{ Затраты на НИОКР } \\
\cline { 2 - 4 } & Россия & Китай & Германия \\
\hline 2017 & 1,1 & 2,1 & 3,0 \\
\hline 2016 & 1,1 & 2,1 & 2,9 \\
\hline 2015 & 1,1 & 2,1 & 2,9 \\
\hline 2010 & 1,1 & 1,7 & 2,7 \\
\hline 2009 & 1,3 & 1,7 & 2,7 \\
\hline 2008 & 1,0 & 1,4 & 2,6 \\
\hline 2007 & 1,1 & 1,4 & 2,4 \\
\hline 2006 & 1,1 & 1,4 & 2,5 \\
\hline
\end{tabular}

Источник. URL: https://knoema.ru/atlas

\footnotetext{
* Миропольский Дмитрий Юрьевич (miropolskiy1959@mail.ru), доктор экономических наук, профессор, СанктПетербургский государственный экономический университет (г. Санкт-Петербург, Россия);

Ломакина Ирина Борисовна (lomakina7311@gmail.com), доктор юридических наук, профессор, Университет Прокуратуры Российской Федерации (Санкт-Петербургский юридический институт) (г. Санкт-Петербург, Россия).
} 
Для наглядности представим динамику удельного веса затрат на НИОКР в ВВП в графической форме (рис. 1-3) ${ }^{1}$.

${ }^{2}$ Миропольский Д.Ю. 2015. Очерки теории продукта: потенциальные формы капитала и плана эпохи до разделения труда. Санкт-Петербург: Издательство Санкт-Петербургского государственного экономического университета.

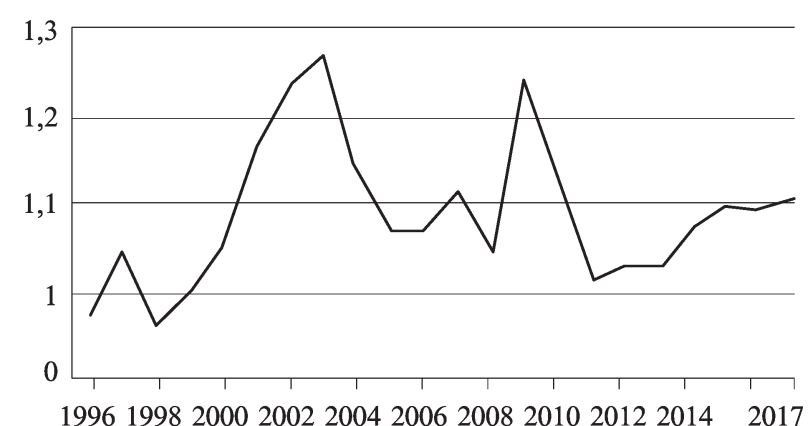

Рис. 1. Динамика затрат на НИОКР в России, $\%$ к ВВП

Источник. URL: https://knoema.ru/atlas

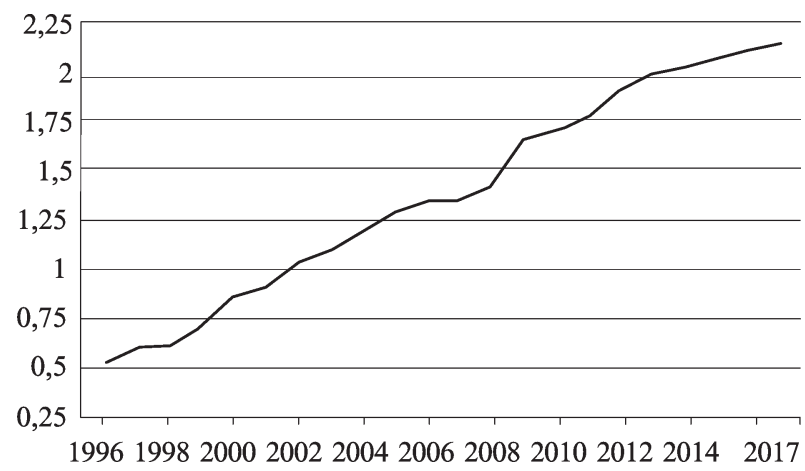

Рис. 2. Динамика затрат на НИОКР в Китае, $\%$ к ВВП

Источник. URL: https://knoema.ru/atlas

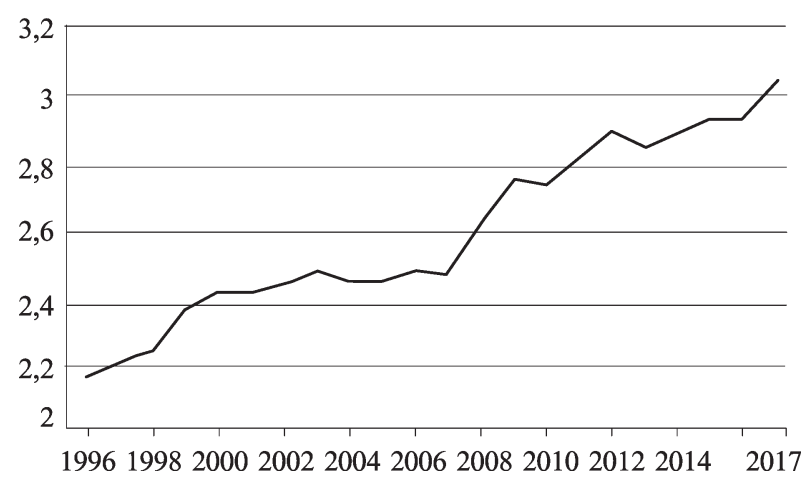

Рис. 3. Динамика затрат на НИОКР в Германии, $\%$ к ВВП

Источник. URL: https://knoema.ru/atlas
Приведенные данные говорят о том, что развитие на инновационной основе в российской экономике в целом находится в неудовлетворительном состоянии. Существуют лишь отдельные области, где исследования и разработки осуществляются успешно. О таком плачевном положении дел говорит не только статистика, но и многочисленные исследования ученых (Аганбегян, 2018. С. 379; Беломестнов, 2019. С. 31; Гринберг, 2019. С. 19).

В данной статье мы хотели бы исследовать проблему перехода к инновационному росту с точки зрения институтов-регуляторов и институтов-стимуляторов и соответствующих эффектов регуляции и стимуляции, возникающих в хозяйственных системах. Для того, чтобы исследовать эффекты регуляции и стимуляции, возникающие именно как системные эффекты, необходимо хозяйственную систему теоретически изобразить.

На рис. 4 на оси абсцисс показаны абстрактные субъекты, осуществляющие определенную экономическую деятельность (S). На оси ординат обозначены результаты хозяйствования каждого субъекта, выраженные в стоимостной форме, или иначе, предельные продукты производства в стоимостной форме $\left(\mathrm{MP}_{1}\right)^{2}$.

На рис. 4 каждый столбик обозначает результат деятельности отдельного экономического субъекта. Видно, что эти результаты последовательно сокращаются. При-

${ }^{2}$ Миропольский Д.Ю. 2015. Очерки теории продукта: потенциальные формы капитала и плана эпохи до разделения труда. Санкт-Петербург: Издательство Санкт-Петербургского государственного экономического университета.

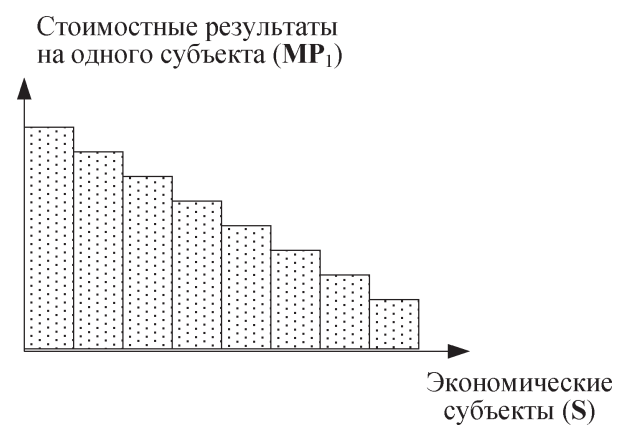

Рис. 4. Результаты деятельности экономических субъектов в хозяйственной системе

Источник. Авторская разработка. 
чины этого сокращения разьясним ниже. На рис. 5 столбиками обозначены предельные затраты тех же субъектов, выраженные в стоимостной форме $\left(\mathrm{MP}_{2}\right)$. Предельные затраты обозначены буквами МР потому, что мы рассматриваем затраты тоже как продукт, но только потребляемый.

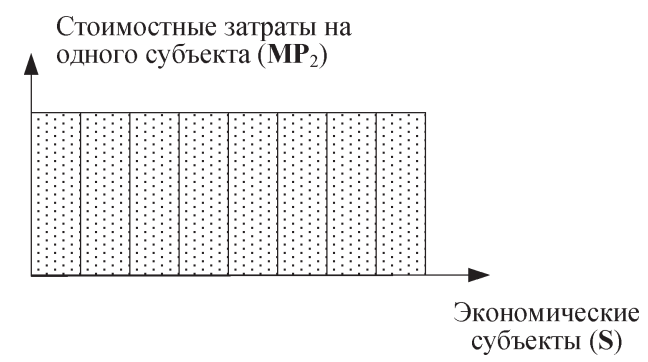

Рис. 5. Затраты экономических субъектов в хозяйственной системе

Источник. Авторская разработка.

Условно предположено, что предельные затраты у всех субъектов одинаковые.

Переменные на осях абсцисс и ординат позволяют объединить рис. 4 и рис. 5 и получить упрощенную модель хозяйственной системы (рис. 6).

Рис. 6 представляет собой простейшую хозяйственную систему потому, что мы определяем ее как процесс производства (результат) и потребления (затраты) продукта 3 . В отличие от рис. 4 и рис. 5, где экономические субъекты представлены конкретными столбиками, на рис. 6 присутствует неопределенное множество таких субъектов.

3 Миропольский Д.Ю. 2015. Очерки теории продукта: потенциальнье формь капитала и плана эпохи до разделения mpуда. Санкт-Петербург: Издательство Санкт-Петербургского государственного экономического университета. С. 11-17.

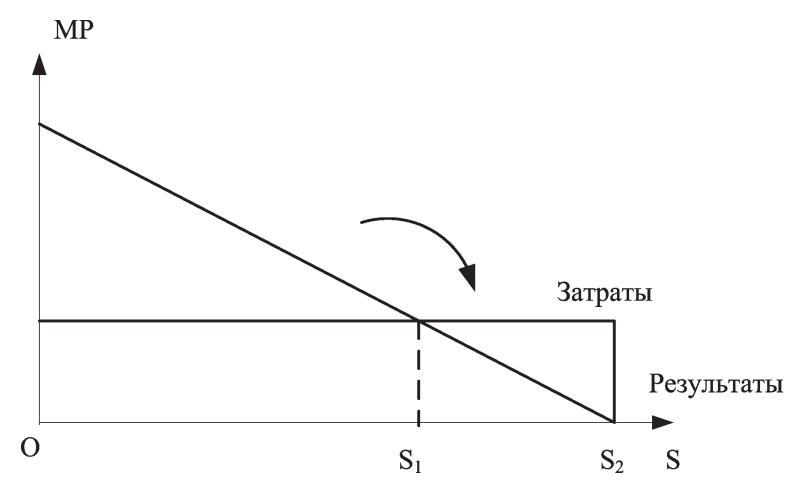

Рис. 6. Упрощенная модель хозяйственной системы

Источник. Авторская разработка.
На рис. 6 видно, что простейшая хозяйственная система распалась на два сектора. Первый сектор (субъекты на отрезке $\mathrm{OS}_{1}$ ) характеризуется тем, что результат в нем выше затрат и, соответственно, есть избыток продукта. Второй сектор (субъекты на отрезке $\mathrm{S}_{1} \mathrm{~S}_{2}$ ) отмечен результатами более низкими, чем затраты. Здесь имеется недостаток продукта.

Основное экономическое содержание первого сектора, продуктоизбыточного, состоит в том, что он производит базовые продукты. Базовые продукты - это продукты необходимого жизнеобеспечения человека, изготавливаемые с высокой производительностью. Отсюда высокие результаты и низкие затраты.

Главный смысл второго сектора, продуктонедостаточного, заключается в создании пионерных продуктов. Это вновь осваиваемые человеком продукты, еще не вошедшие в комплекс необходимого жизнеобеспечения. Как следствие - высокие затраты и низкие результаты. Деление продуктов на базовые и пионерные объясняет снижающиеся результаты хозяйственной деятельности субъектов $\mathrm{S}$ на рис. 4: По мере движения вправо по оси абсцисс экономические субъекты производят все менее базовые продукты и, соответственно, все более пионерные. При одинаковых затратах (рис. 5) это дает все меньший результат.

Мы получили хозяйственную систему, включающую базовый и пионерный секторы. Вся сфера НИОКР является важнейшей составляющей пионерного сектора. В настоящей статье, для простоты, мы вообще отождествим пионерный сектор и сферу НИОКР.

Модель на рис. 6 показывает, что пионерный сектор (a, значит, вся сфера НИОКР) не может развиваться без перераспределения избыточного продукта базового сектора. Возникает вопрос, какой экономический механизм должен осуществлять это перераспределение?

Существует два крайних варианта перераспределения избыточного базового продукта в пионерный сектор. Первый вариант представлен на рис. 7.

На рис. 7 прямоугольник OKLF демонстрирует предельные выручки экономичес- 


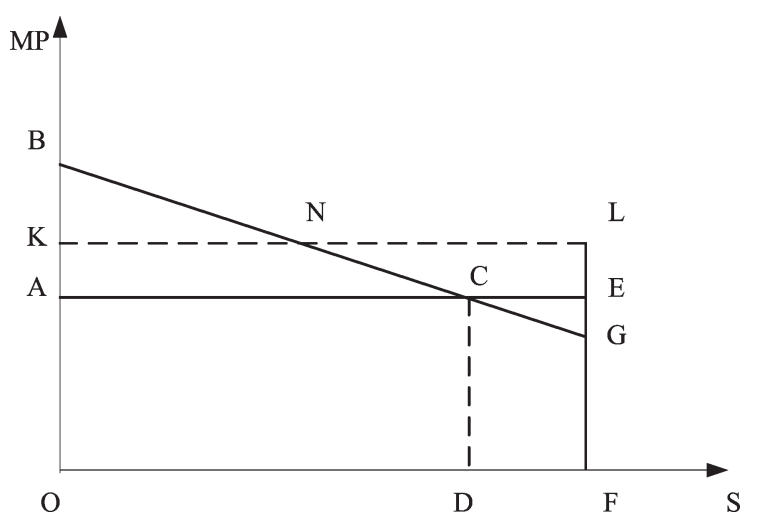

Рис. 7. Рыночная хозяйственная система

Источник. Авторская разработка.

ких субъектов S. Мы видим, что выручка каждого субъекта превышает затраты (OAEF) и дает каждому субъекту одинаковую прибыль (AKLE). Иначе говоря, каждый экономический субъект в данной хозяйственной системе может продать продукцию по приемлемым ценам и реализовать себя как частный собственник.

Это возможно при наличии двух условий. Во-первых, и это главное, избыток продукта базового сектора существенно больше нехватки продукта в пионерном секторе ( $\Delta$ ABC $>\Delta$ CEG, рис. 7). Данное обстоятельство позволяет перераспределить из базового сектора объем ресурсов, достаточный для обеспечения запросов пионерного сектора $(\Delta \mathrm{KBN}=\Delta \mathrm{NLG})$, и одновременно сохранить в базовом секторе ресурсы, достаточные для получения субъектами базового сектора нормальной прибыли.

Во-вторых, рыночный вариант инновационного развития возможен, если субъекты базового сектора согласятся продать свою продукцию по заниженным ценам, а продукцию базового купить по завышенным.

Если оба условия выполнены, мы видим, что ресурсы базового сектора посредством рынка и, прежде всего рыночных цен, в размере KBN перераспределялись на цели инновационного развития данной экономики.

Теперь посмотрим на ситуацию, изображенную посредством рис. 8 .

На рис. 8 нехватка ресурсов в пионерном секторе, даже в части затрат, столь огромна ( $\triangle \mathrm{CEF})$, что в базовом секторе просто не хватит избыточного продукта, что-

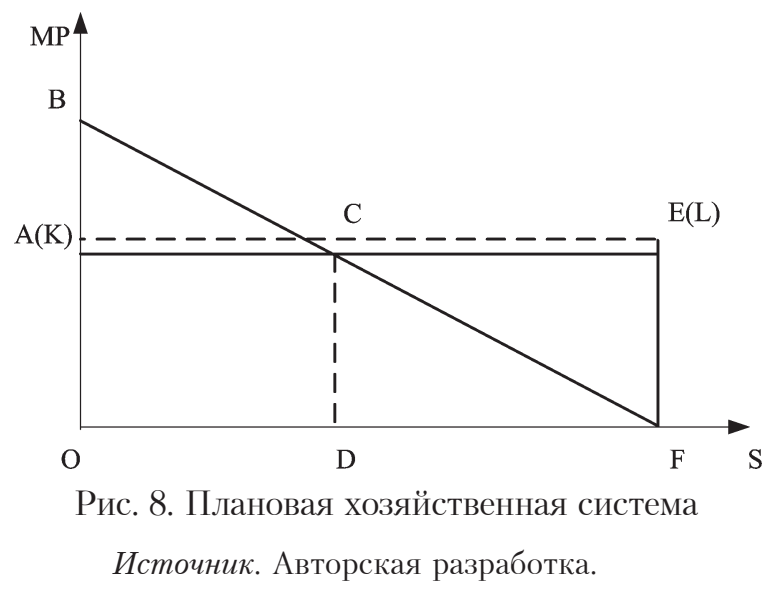

бы покрыть потребности пионерного сектора и еще обеспечить всем нормальную прибыль. При подобных условиях, если субъекты этой экономики попытаются торговать, то цены будут такими, что выручки сравняются с затратами: OAEF=OKLF. B данной хозяйственной системе перераспределение избыточного продукта базового сектора в пользу пионерного возможно только на основе плана.

Фундаментальная причина, по которой в одном случае инновационное развитие возможно на базе рынка, а в другом - на базе плана, заключается в пропорции, складывающейся между базовым и пионерным секторами экономики ${ }^{4}$.

Теперь, когда мы получили основные разновидности хозяйственной системы рыночную и плановую, можно приступить к анализу эффектов регуляции и стимуляции.

\section{Эффекты регулящии и стимулящии}

Рассмотрим сначала эффекты регуляции и стимуляции в чистом виде. Начнем с эффекта регуляции (рис. 9).

На рис. 9а изображена модель рыночной экономики. Для простоты пунктирная линия, фиксирующая выручку, убрана. Признаком рыночной экономики является маленький пионерный сектор, по сравнению с базовым. Допустим, что сообщество людей, создавших данную рыночную экономику, ощущает необходимость резкого увеличения пионерного сектора. Однако институты рынка не по-

${ }^{4}$ Максимцев И.А., Миропольский Д.Ю., Тарасевич Л.С. (Ред.) 2016. Евразийская политическая экономия: учебник. Санкт-Петербург: Издательство Санкт-Петербургского государственного экономического университета. С. 220-224. 


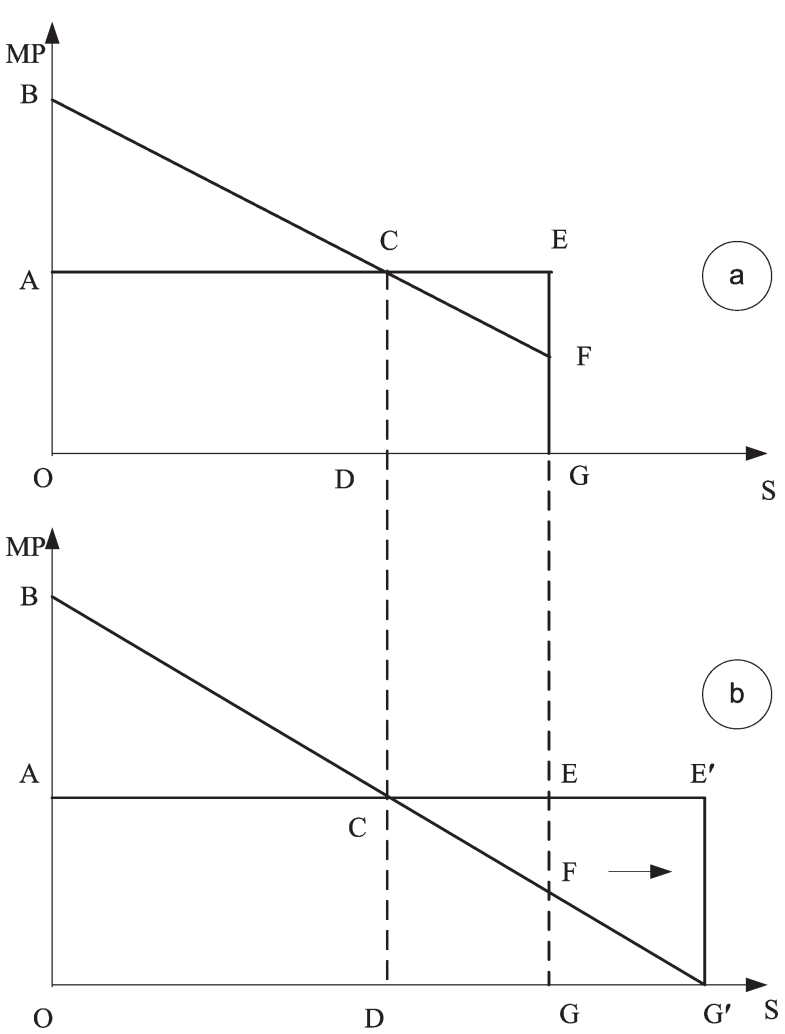

Рис. 9. Эффект регуляции

Источник. Авторская разработка.

зволят этого сделать. При наличии базового сектора, представленного на рис. 9а, увеличение пионерного сектора будет снижать прибыли частных собственников. Тогда указанное сообщество переходит к плановой экономике (рис. 9b). В плановой экономике прибыль индивидуальных частных собственников перестает играть свою роль в связи с отсутствием индивидуальных частных собственников. Это позволяет отрегулировать величину пионерного сектора путем плановой мобилизации на его нужды всех избыточных ресурсов базового сектора. На рис. $9 \mathrm{~b}$ приращение величины пионерного сектора на величину GEE'G' и есть эффект регуляции.

При этом следует учесть, что величина GEE'G' это положительный эффект регуляции, возникающий при переходе от рынка к плану. Если идти от рис. 9b к риc. 9a, то возникнет отрицательный эффект регуляции, связанный с ликвидацией пионерного сектора на величину GEE'G' при переходе от плана к рынку.

Теперь рассмотрим эффект стимуляции. Тоже в чистом виде (рис. 10).
На рис. 10а представлена плановая экономика: пионерный сектор по отношению к базовому большой. В итоге торговля невозможна, ибо пунктирная линия выручки (которая здесь не показана) сольется с затратами. Допустим, люди, создавшие данную экономику, начинают ощущать, что затраты в их экономике слишком высокие, а результаты ниже, чем могли бы быть. Такое соотношение затрат и результатов возникло в силу того, что хозяйственные субъекты в плановой экономике не имеют должных стимулов к эффективной работе. Тогда данное людское сообщество осуществляет переход к рынку, где каждый индивидуально отвечает за свои затраты и результаты. Возникшая рыночная экономика представлена на рис. 10b. Мы видим, что линия AЕ, обозначавшая уровень затрат в бывшей плановой экономике, сместилась в положение A'E', то есть затраты снизились. А линия BF, которая раньше показывала уровень производительности, теперь находится в положении B'F': производительность субъектов выросла. В итоге пионерный сектор сжался, а базовый вырос. Снижение

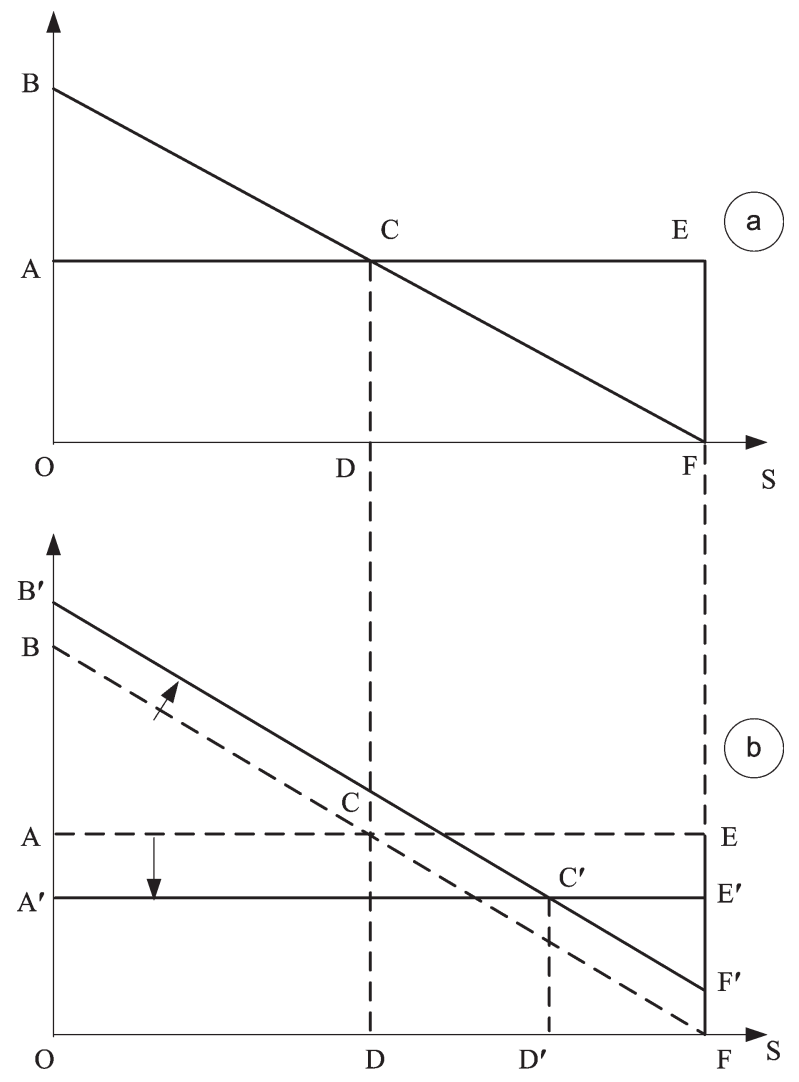

Рис. 10. Эффект стимуляции

Источник. Авторская разработка. 
затрат на общую величину A'AEE' и рост результатов на величину BB'F'F и есть эффект стимуляции.

При этом, как и в случае с эффектом регуляции, при переходе от плана к рынку мы имеем положительный эффект стимуляции. Если же мы перейдем от рынка к плану (от рис. 10b к рис. 10a), эффект стимуляции примет отрицательное значение.

От рассмотрения эффектов регуляции и стимуляции в чистом виде необходимо перейти к их изучению в ситуации, более приближенной к реальности.

\section{Инновационный процесс и конкретные комбинации әффектов регулящии и стимуляции}

Е. Гайдар (2006), критикуя советскую экономику, в свое время писал: «Советский союз добывал в 8 раз болыше железной руды, чем США, выплавлял из этой руды втрое больше чугуна, стали из этого чугуна - вдвое больше. Машин из этого металла производил по стоимости примерно столько же, сколько США ... В расчете на единицу конечного продукта СССР расходовал в 1980 г. стали в 1,8 раз больше, чем США, цемента в 2,3 раза, минеральных удобрений в 7,6 раз, лесопродуктов - в 1,5 раза».

Допустим, что цифры, приводимые Е. Гайдаром, достоверно отражают положение дел. Допустим также, что приведенные Е. Гайдаром данные не связаны с исторически накопленной материально-технической базой СССР и обусловлены исключительно институтами плана. Однако даже в этом случае критика Гайдара односторонне выдергивает из контекста отрицательный эффект стимуляции и игнорирует положительный эффект регуляции, присущий плановой экономике. Проблема заключается в том, что любой хозяйственной системе всегда присуще определенное сочетание эффектов регуляции и стимуляции и ее эффективность всегда определяется этим конкретным сочетанием. А если это так, то нам и надо перейти к изучению таких сочетаний (рис. 11).

На рис. 11а изображена плановая экономика. Эта плановая экономика перешла к рынку (рис. 11b). Как следствие, возник отрицательный эффект регуляции, привед-

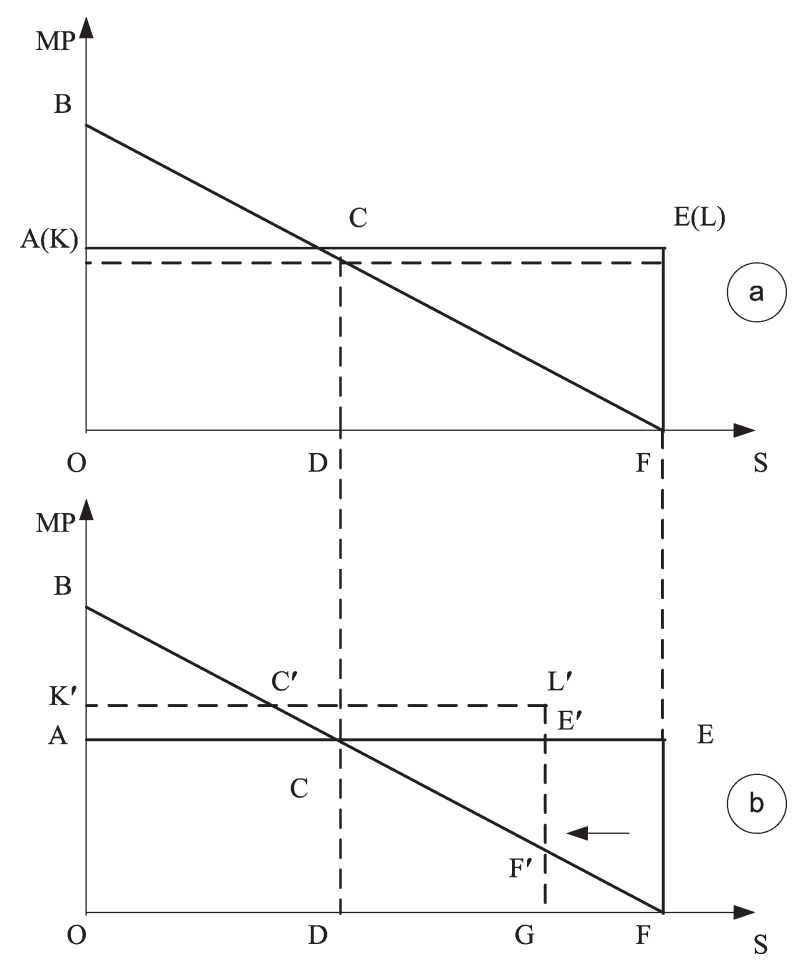

Рис. 11. Отрицательный эффект регуляции при переходе от плана к рынку

Источник. Авторская разработка.

ший к свертыванию ряда пионерных производств (GE'EF). В результате этого свертывания у оставшихся субъектов базового и пионерного секторов возникла прибыль (AK'L'E'), обеспеченная избыточным продуктом базового сектора (АВС).

Однако переход от плана к рынку сопровождается не только отрицательным эффектом регуляции, но и положительным эффектом стимуляции (рис. 12).

Рис. 12a просто повторяет рис. 11b и показывает отрицательный эффект регуляции. Положительный эффект стимуляции отображен на рис. $12 \mathrm{~b}$.

Для упрощения рассуждений мы исходим из того, что в рассматриваемой экономике эффект стимуляции повлиял только на результат и не затронул затраты. Это выразилось в том, что на рис. $12 \mathrm{~b}$ линия BF', обозначающая уровень производительности субъектов экономики, сместилась в положение B'F”. Общий прирост продукции за счет эффекта стимуляции составил величину BB2 F"F', а прибыли возросли на величину K'K”'L'L'.

Соотнесем отрицательный эффект регуляции и положительный стимуляции. 


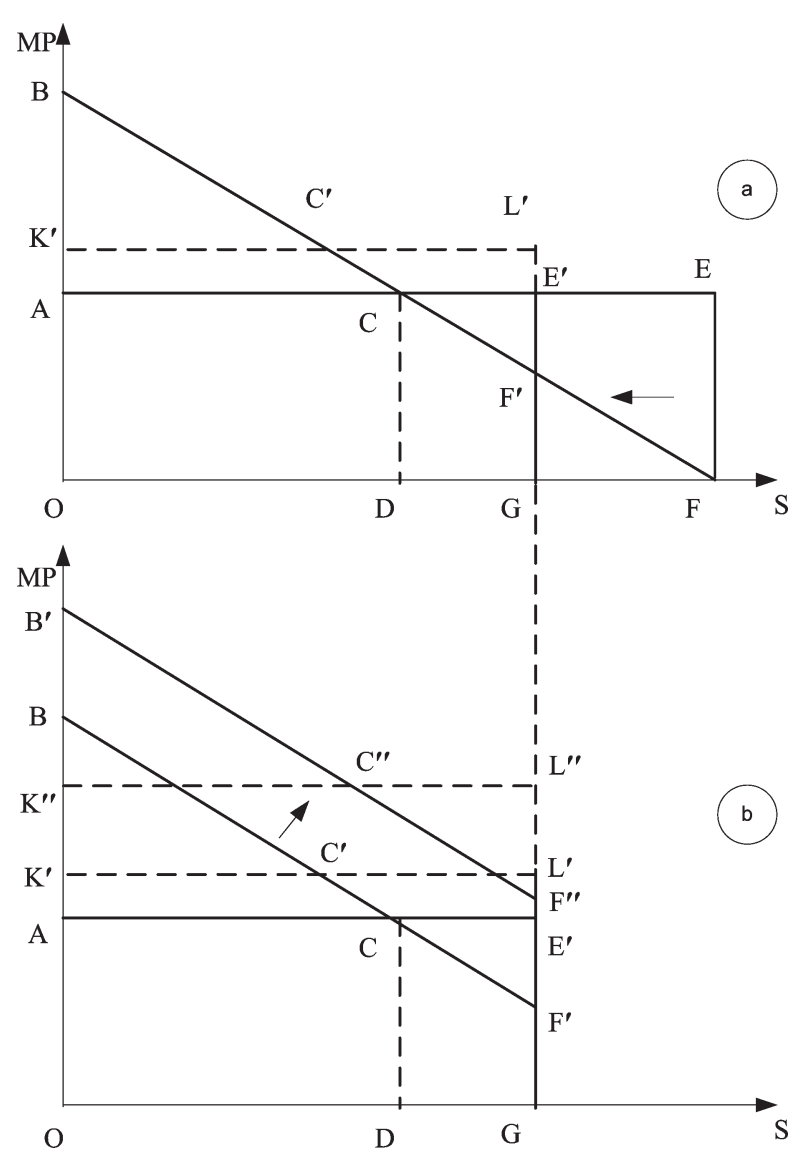

Рис. 12. Положительный эффект стимуляции при переходе от плана к рынку

Источник. Авторская разработка.

Отрицательный эффект регуляции сократил производство на величину GE'EF (риc. 12a). Положительный эффект стимуляции увеличил производство на величину ВВ' F'F' (рис. 12b). Невооруженным взглядом видно, что BB'F'F'> GE'EF. То есть, в нашем примере положительный эффект стимуляции перекрыл отрицательный эффект регуляции и, в результате, общество, перейдя к рынку, получило в свое распоряжение больше ресурсов.

Как распорядилось условное сообщество возросшим количеством продуктов? Посмотрим на рис. 13. Рис. 13а показывает ситуацию сложения эффектов регуляции и стимуляции, а рис. 13b демонстрирует события, развернувшиеся далее.

Мы видим, что новоявленные бизнесмены, во-первых, возродили омертвелую часть пионерного сектора экономики (GL ${ }_{2}{ }^{\prime}{ }_{2}, ' G$ '), рис. 13b). Причем, обратите внимание, что за счет эффекта стимуляции

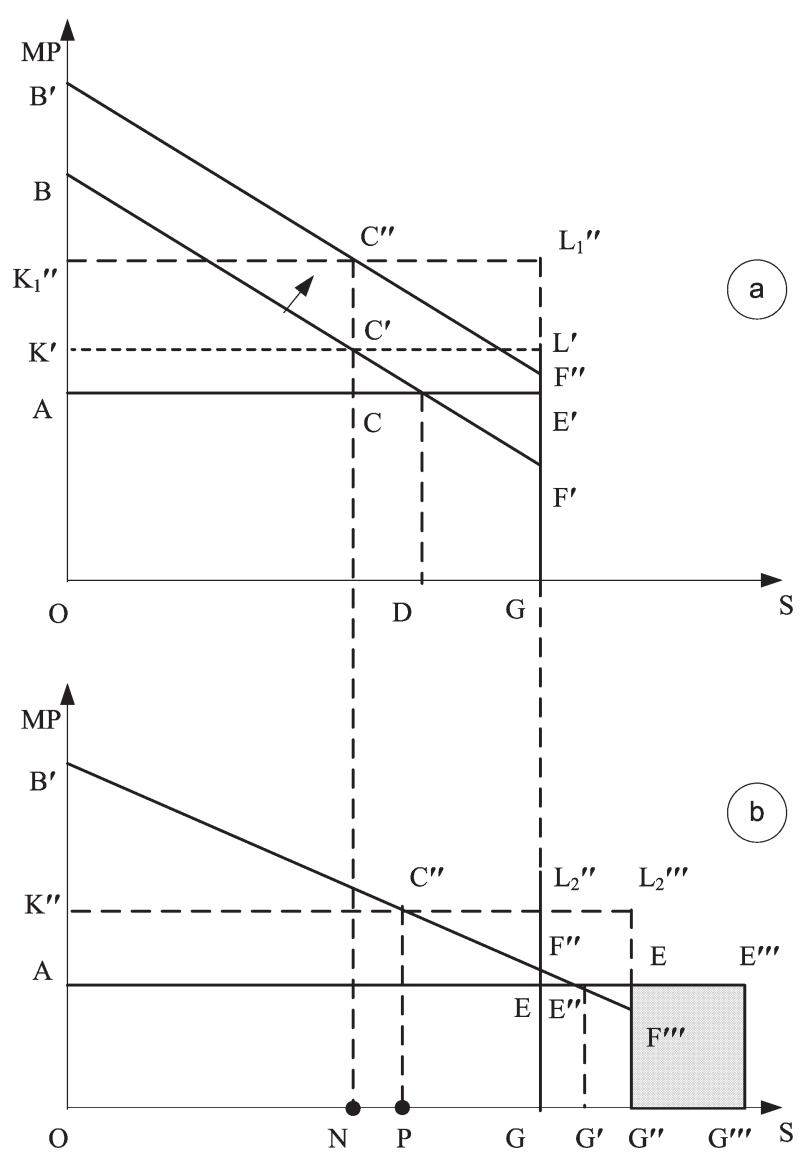

Рис. 13. Переход к инновационному типу развития на основе эффекта стимуляции

Источник. Авторская разработка.

у части субъектов пионерного сектора стоимость результата (GF”'E”G') стала превышать стоимость затрат (GE'E”'G'). Во-вторых, бизнесмены вложили часть прибыли в новые пионерные производства в размеpe G"EE”'G”' (рис. 13b). Это пока лишь вложения без какой-либо продукции. Но самое главное, что в рыночной экономике пионерный сектор, воплощающий инновационное развитие, увеличился!

Для наглядности сравним новую рыночную ситуацию в экономике с исходной, плановой (рис. 14). На рис. 14а изображена новая рыночная система, на рис. $14 \mathrm{~b}-$ старая плановая. Сравнение явно не в пользу плановой.

Эффект стимуляции, перекрывающий отрицательный эффект регуляции, привел к тому, что, во-первых, общий выпуск при тех же затратах в рыночной экономике больше, чем в плановой (OB'F'"G" > OBF, рис. $14 \mathrm{a}, 14 \mathrm{~b})$, т. е. имеет место экономи- 
ческий рост. Во-вторых, часть производств, бывших пионерными в условиях плана, повысили свою эффективность настолько, что стали базовыми. В-третьих, возник новый сегмент пионерного сектора, связанный с новейшими технологиями, которые знаменуют начало развития экономики на инновационной основе и которых не было в плановом хозяйстве.

В связи со всем этим, модель, представленную на рис. 14а, можно условно назвать «мечта восточноевропейского демократа». И действительно, если вспомнить мечты конца 80-х - начала 90-х, то среди демократической общественности постоянно витали идеи о том, как частная собственность возродит в людях чувство хозяина и создаст новые стимулы к труду. Правда, система, изображенная посредством риc. $14 \mathrm{a}$, работает не только за счет мощного положительного эффекта стимуляции. Должен быть исполнен еще ряд условий. Вернемся к рис. 13. Следует обратить внимание, что реанимация омертвевшего пионерного сектора (GL, ${ }_{2} \mathrm{~L}_{2}$ ”'G”, рис. $\left.13 \mathrm{~b}\right)$ согласно логике представленной двухсекторной модели необходимо ведет к снижению об-

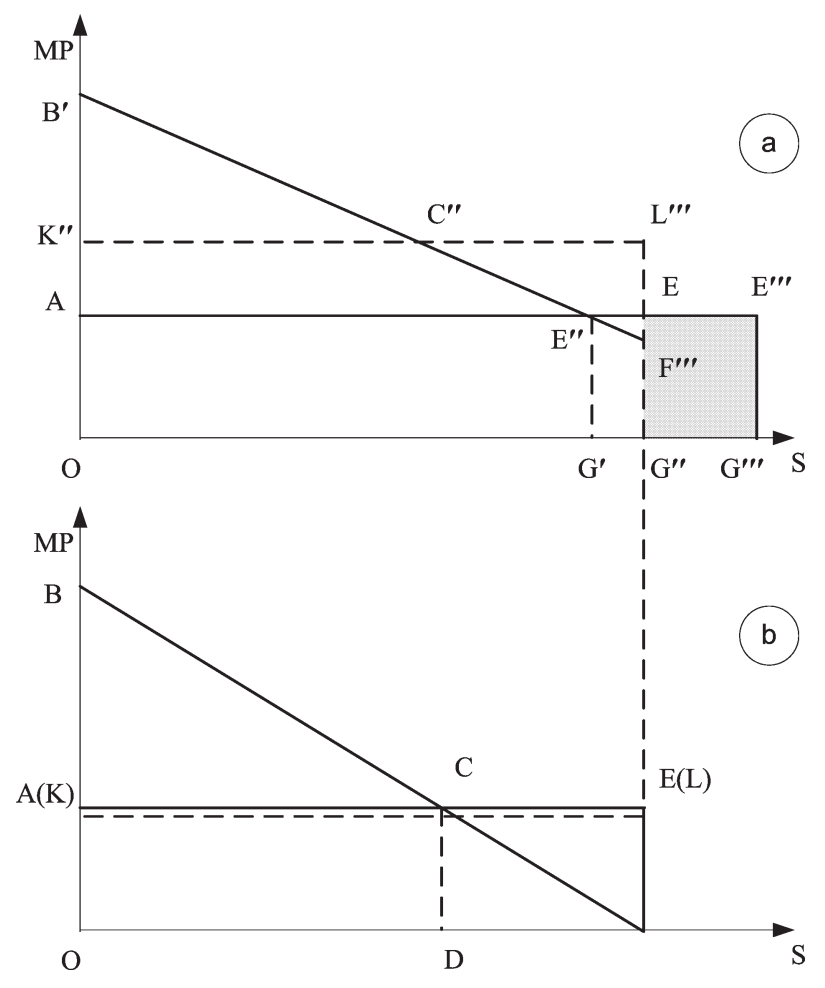

Рис. 14. Возникшая рыночная экономика и исходная плановая

Источник. Авторская разработка. щего уровня прибыльности в экономике: линия $\mathrm{K}_{1}{ }^{\prime} \mathrm{L}_{1}$ " на рис. 13а располагается выше, чем линия $\mathrm{K}_{2}{ }^{\prime} \mathrm{L}_{2}$ ” на рис. $13 \mathrm{~b}$. Кроме того, из снизившейся прибыли бизнесмены вложили изрядные деньги в новейшие технологии (G”EE”' G”'). Вернутся ли эти вложения, да еще и с прибылью - большой большой вопрос.

Поэтому экономическая модель на риc. 14а осуществима, если бизнесмены

a) не поддадутся искушению вложить деньги не в пионерный, а в базовый сектор, где прибыли выше и надежнее;

б) не захотят вложить полученные прибыли в финансовые спекуляции;

в) не вывезут прибыли за рубеж, в том числе в оффшорные зоны;

г) выдержат агрессивную внешнюю конкуренцию в сфере новейших технологий.

Однако данные факторы, осложняющие действие эффекта стимуляции, мы в статье специально не рассматриваем.

Теперь предлагаем очнуться от мечтаний и еще больше приблизиться к реальности. Посмотрим, как шел переход от плана к рынку в России и как сочетались в процессе этого перехода эффекты регуляции и стимуляции (рис. 15).

На рис. 15а изображены условные Соединенные Штаты Америки. В США рыночная экономика. На рис. 15а это отражено посредством соответствующей пропорции между базовым и пионерным секторами и вытекающего из нее уровня прибылей (AKLE). На рис. 15b представлена условная экономика Советского Союза. Советский Союз, конкурируя с США, должен был иметь примерно такой же пионерный сектор, как и США. Однако, как видно на рис. $15 \mathrm{~b}$ он даже больше (DCEF $\left.<\mathrm{D}_{1} \mathrm{C}_{1} \mathrm{E}_{1} \mathrm{~F}_{1}\right)$. Это связано с тем, что отрицательный эффект стимуляции приводил к более низкой производительности в СССР (затраты мы условно принимаем равными). На рис. $15 \mathrm{~b}$ относительно низкая производительность отражена смещением линии $\mathrm{B}_{1} \mathrm{~F}_{1}$ влево по сравнению с линией $\mathrm{BF}$ на рис. 15а. Относительно более низкая производительность делала в СССР пионерными те производства, которые в США были уже базовыми. Содержать более громоздкий пионерный сектор при более скудных ресурсах базово- 


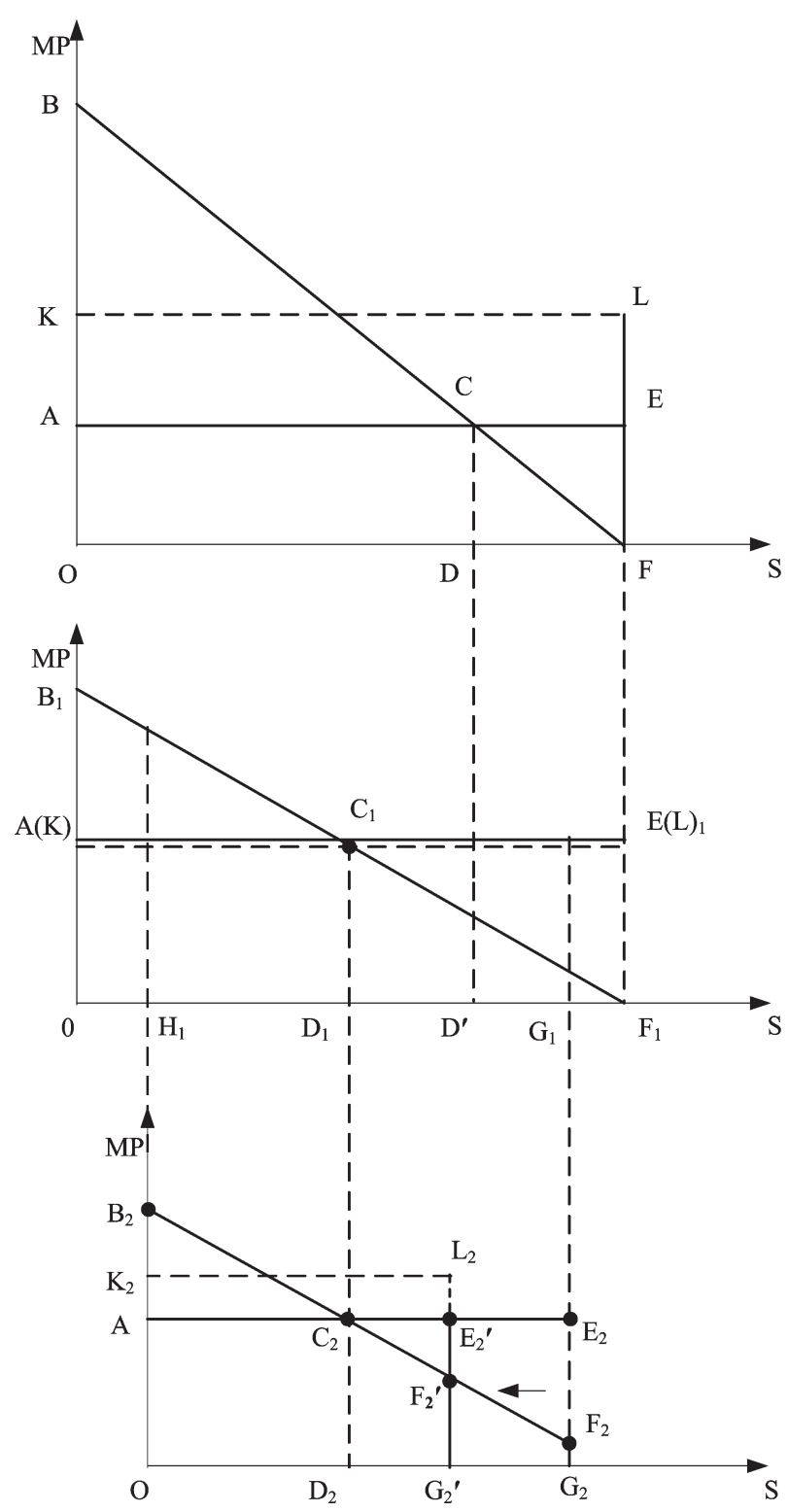

Рис. 15. Эффекты регуляции и стимуляции при переходе России к рынку

Источник. Авторская разработка.

го $\left(\mathrm{A}_{1} \mathrm{~B}_{1} \mathrm{C}_{1}<\mathrm{ABC}\right.$, рис. $15 \mathrm{~b}$, рис. $\left.15 \mathrm{a}\right)$ можно лишь в условиях плановой экономики.

Распад СССР и превращение России в самостоятельное государство условно показаны на рис. 15с путем отпадения экономических субъектов на отрезках $\mathrm{OH}_{1}$ (рис. 15b) и $\mathrm{G}_{1} \mathrm{~F}_{1}$ (рис. 15b). Приватизация и освобождение цен в России породили отрицательный эффект регуляции, что привело к сжатию пионерного сектора $\left(\mathrm{D}_{2} \mathrm{C}_{2} \mathrm{E}_{2} \mathrm{G}_{2}>\mathrm{D}_{2} \mathrm{C}_{2} \mathrm{E}_{2}{ }^{\prime} \mathrm{G}_{2}{ }_{2}\right.$, рис. 15c).

Что могло нейтрализовать отрицательный эффект регуляции? Только положительный эффект стимуляции, перекрываю- щий его, как показано на рис. 12b. Тогда линия $\mathrm{B}_{2} \mathrm{~F}_{2}$ на рис. 15 с сместилась бы вправо, предоставив новым российским бизнесменам ресурсы для реанимации умирающего пионерного сектора. Однако вся драма российского перехода к рынку состоит в том, что никакого существенного эффекта стимуляции не возникло. На рис. $15 \mathrm{c}$ он для простоты приравнен к нулю. Поэтому на графике виден только отрицательный эффект регуляции, сжимающий пионерский сектор.

Итогом такой комбинации эффектов регуляции и стимуляции стала потеря существенной части высокотехнологичных отраслей и специализация России на нефти и газе.

Резким контрастом российского варианта перехода к инновационному развитию на основе рынка является Китай. Можно предположить, что в Китае движение к рынку порождало отрицательный эффект регуляции, однако он с лихвой перекрывался положительным эффектом стимуляции. Это давало в руки, как бизнесу, так и бюрократии, дополнительные и существенные ресурсы для инновационного развития. Известно, что китайские реформы начались с перевода крестьян на подворный подряд. Однако даже столь осторожный шаг в сторону рынка породил мощный положительный эффект стимуляции. В Китае с 1979 по 1985 г. доходная часть бюджета выросла на $64 \%$. Доля сельского хозяйства в этом приросте была в 2,8 раза выше, чем в промышленности (Гельбрас, 1990. С. 57).

Почему один народ реагирует на рыночные стимулы взлетом трудовой активности, а другой либо не реагирует, либо реагирует неадекватно? Это слишком серьезный вопрос, чтобы отвечать на него в двух словах. Он требует отдельного исследования.

В заключение приведем данные по удельному весу затрат на НИОКР в ВВП в Беларуси, аналогичные представленным в начале статьи по России, Китаю и Германии (табл. 2).

В графической форме динамика удельного веса затрат на НИОКР выглядит следующим образом (рис. 16).

Данные табл. 2 и рис. 16 позволяют предположить, что ситуация с инновационным развитием в Беларуси ближе к си- 
Таблица 2

Затраты на НИОКР в Беларуси, \% ВВП

\begin{tabular}{|c|c|}
\hline Год & Затраты на НИОКР \\
\hline 2017 & 0,6 \\
\hline 2016 & 0,5 \\
\hline 2015 & 0,5 \\
\hline 2010 & 0,7 \\
\hline 2009 & 0,6 \\
\hline 2008 & 0,7 \\
\hline 2007 & 1,0 \\
\hline 2006 & 0,7 \\
\hline
\end{tabular}

Источник. URL: https://knoema.ru/atlas

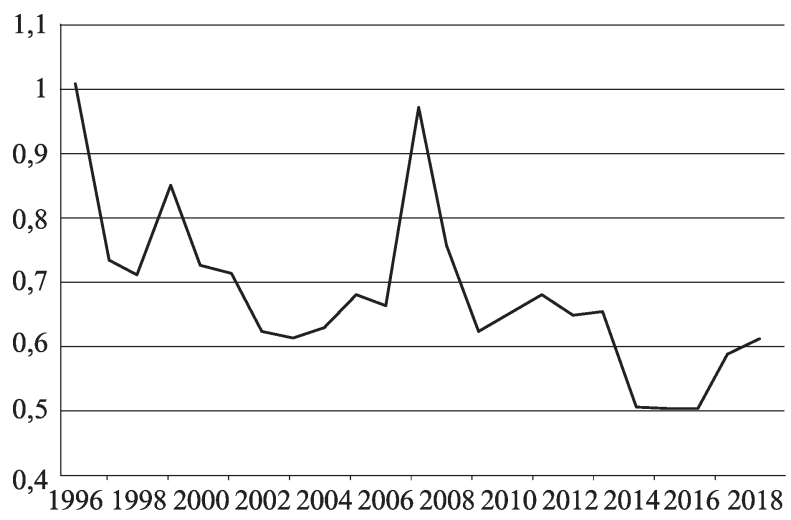

Рис. 16. Динамика затрат на НИОКР в Беларуси, $\%$ к ВВП

Источник. https://knoema.ru/atlas

туации в России, чем в Китае или в Германии. Следовательно, логично также предположить, что существующая хозяйственная система Беларуси не обеспечивает должного инновационного развития. При этом, хозяйственная система Беларуси представляет собой определенную комбинацию плана и рынка. Значит, для перехода экономики Беларуси на инновационные рельсы эту комбинацию надо изменить либо в сторону усиления рыночных начал, либо в сторону усиления плановых. Возникает вопрос, как, конкретно в Беларуси, то или иное изменение соотношения институтов рынка и плана изменит соотношение эффектов стимуляции и регуляции? Как следует из изложенного выше, конкретная комби- нация этих эффектов создает главную предпосылку либо для инновационного развития, либо для его отсутствия.

\section{СПИСОК ЛИТЕРАТУРЫ (REFERENCES)}

Аганбегян А.Г. 2018. Финансы, бюджет и банки в новой России. Москва: Дело. [Aganbegyan A.G. 2018. Finance, budgets and banks in the new Russia. Moscow: Delo. (In Russ.)]

Беломестнов В.Г. 2019. Инновационная экономика России: институциональный подход. Проблемы современной экономики. № 4. С. 30-34. [Belomestnov V.G. 2019. Innovative economy in Russia: Institutional approach. Problemy Souremennoy Ekonomiki. No 4. PP. 30-34. (In Russ.)]

Бодрунов С.Д. 2018. Ноономика. Москва: Культурная революция. 432 с. [Bodrunov S.D. 2018. Noonomics. Moscow: Kul'turnaya Revolyutsiya. 432 p. (In Russ.)]

Гайдар E.T. 2006. Гибель империи. Уроки для современной России. Москва: Российская политическая энциклопедия. [Gaydar E.T. 2006. Collapse of an Empire: Lessons for Modern Russia. Moscow: Rossiyskaya politicheskaya entsiklopediya. (In Russ.)]

Гельбрас В.Г. 1990. Экономическая реформа в КНР. Очерки, наблюдения, размышления. Москва: Международные отношения. [Gel'bras V.G. 1990. Economic reform in China. Essays, observations, reflections. Moscow: Mezhdunarodnye otnosheniya. (In Russ.)]

Глазьев С.Ю. 2016. Экономика будущего. Есть ли у России шанс? Москва: Книжный мир. [Glaz'ev S.Y. 2016. The economy of the future. Does Russia have a chance? Moscow: Knizhnyy mir. (In Russ.)]

Гринберг Р.С. 2019.Технологические революции и социум: мировой тренд и российская специфика. Экономическое возрождение России. № 1. C. 17-22. [Grinberg R.S. 2019. Technological revolutions and the society: Global trend and russian specificity. Ekonomicheskoe vozrozhdenie Rossii. No 1. PP.17-22. (In Russ.)]

Шульце П.В. 2017. Будущее промышленности: четвертая революция - функции государства и общества. Экономическое возрождение России. № 2. C. 39-46. [Schulze P.W. 2017. Future of industry: The fourth revolution - functions of state and society. Ekonomicheskoe vozrozhdenie Rossii. No 2. PP. 39-46. (In Russ.)] 
In citation: Belorusskiy Economicheskiy zhurnal. 2020. No 2. PP. 132-142.

Belarusian Economic Journal. 2020. No 2. PP. 132-142.

\title{
REGULATION AND STIMULATION EFFECTS: INSTITUTIONAL MECHANISMS OF FORMATION AND ROLE IN INNOVATIVE DEVELOPMENT OF ECONOMIC SYSTEMS
}

\author{
Dmitriy Miropolskiy ${ }^{1}$, Irina Lomakina ${ }^{2}$ \\ Author affiliation: ${ }^{1}$ St. Petersburg State Economic University (St. Petersburg, Russia); \\ ${ }^{2}$ University of the Prosecutor's Office of the Russian Federation (St. Petersburg, \\ Russia). \\ Corresponding author: Dmitriy Miropolskiy (miropolskiy1959@mail.ru).
}

ABSTRACT. There has been researched the impact of plan andmarket institutes on forming regulation and stimulation effects based on applying a two-sector models of the economic system. The importance of combining positive and negative values of these effects in specific conditions of a particular national economy is substantiated for innovative development. The effects of moving from plan to market and from market to plan are illustrated.

KEYWORDS: plan institutes, market institutes, regulation effect, stimulation effect, innovative development.

JEL-code: A10, E02, E11.

DOI: $10.46782 / 1818-4510-2020-2-132-142$

Received 24.04.2020 